\title{
A sliding mode contact electrification based (4) triboelectric-electromagnetic hybrid generator for small-scale biomechanical energy harvesting
}

Venkateswaran Vivekananthan, Woo Joong Kim, Nagamalleswara Rao Alluri, Yuvasree Purusothaman, K. S. Abisegapriyan and Sang-Jae Kim*

\begin{abstract}
The present work describes the hybridization of two different energy harvesters works simultaneously in a single package. By applying simultaneous mechanical force, two components such as triboelectric nanogenerator (TENG) and electromagnetic generator (EMG) independently produce power. The hybrid device was made with a polymeric cylinder composed of Kapton in the inner wall; a copper coil wound outside the cylinder and neodymium magnet and small bits of paper housed inside it. The paper flakes having the dimension of $5 \mathrm{~mm} \times 5 \mathrm{~mm}$, which are triboelectric positive slides over the negative triboelectric layer Kapton. The potential difference between the two different triboelectric material leads to the generation of electric power. The triboelectric component generates the maximum output with the voltage of $\approx 20 \mathrm{~V}$ and the current of $300 \mathrm{nA}$. The magnet inside the cylinder moves simultaneously along with the paper made the production of electric flux in the coil. The alternating magnetic flux induces the current in the outer coil as per the Lenz's law. The maximum output generated from the EMG component with the obtained voltage of $2 \mathrm{~V}$ and the maximum current of $10 \mathrm{~mA}$. Further, to analyze the actual working behavior of the device, commercial capacitor charging behavior was analyzed. The TENG component runs the consistent charging behavior, whereas the EMG component offers a rapid charging behavior, under hybrid mode both the merits can be utilized. The device has had placed in a backpack, and the biomechanical energy from human motions such as walking, running and jumping had been demonstrated. This study confirms that the proposed hybrid generator is capable of powering small electronic devices such as global positioning system (GPS), flashlights and potentially be able to use as an active MEMS/NEMS-based self-powered sensor.
\end{abstract}

Keywords: Biomechanical energy, Triboelectric, Electromagnetic, Hybrid generator

\section{Introduction}

A vast amount of vibration energy had been produced in the environment from our day to life either naturally or by human-made [1]. This vibration energy can be found in the form of vehicle motions [2], human motions [3], ocean waves [4], wind [5], and heavy industries [6]. After the invention of nanogenerators, it has been reported that the waste mechanical energy can be scavenged and eventually been used for powering up small electronic devices

\footnotetext{
*Correspondence: kimsangj@jejunu.ac.kr

Nanomaterials and Systems Laboratory, Department of Mechatronics Engineering, Jeju National University, Engineering Building No:-4, D-130, Ara-1-Dong, Jeju-Si, Jeju-Do 63243, South Korea
}

and can also be used for portable and wearable applications $[7,8]$. By using the nanogenerators, the vibration energy can be scavenged using piezoelectric nanogenerator (PENG) [9], triboelectric nanogenerator (TENG) [10], and electromagnetic generator (EMG) [11]. Among these effects, TENG has shown enormous advantages such as simple fabrication, low cost, and lightweight [12]. Other than harvesting vibration energy TENGs can be used to harvest most of the forms of mechanical energy such as wind [13], ocean [14], rotational [15], tidal [16] and acoustic [17]. On the other hand, TENG itself cannot produce high current to use for various real-time applications. To improve the efficiency of the TENG device, a hybrid approach has been utilized with the combination 
of two different energy harvesting modes in a single package. This type of hybrid nanogenerators can be used for boosting the electrical output performance of the nanogenerator and can also be able to improve the efficiency of the energy harvester. Among the various hybrid energy harvesters, TENG and EMG hybrid combination had gained huge attention because of its energy harvesting performance under the simultaneous mechanical motions $[18,19]$. TENG and EMG can work efficiently as a hybrid generator under sliding, contact-separation and rotation modes.

Very recently many researchers are working on hybrid generators for scavenging vibrational energy for various self-powered applications. He et al. had reported a hybrid generator composed of TENG, PENG, and EMG in a single unit for self-powered wireless monitoring system [20]. Similarly, Shao et al. fabricated a hybrid generator device with TENG and EMG components for scavenging water wave energy with the sea wave oscillation and eventually been used to operate an electronic thermometer [21]. In either case, the device uses a complex construction and uses expensive triboelectric materials such as copper, carbon nanotubes, polyethylene terephthalate (PET). However, the device used in the present manuscript is used as the triboelectric material which is very cheap and simple in overall construction of the device. Since it is small cylindrical in shape it can easily harvest vibrational motions upon any kind of mechanical motions. The triboelectric components works on sliding mode with paper as triboelectric material which cannot create damage on the Kapton layer due to friction like the traditional sliding mode triboelectric nanogenerator. The device the have a smaller dimension which has the dimension equal to $\mathrm{C}$ size battery and houses both triboelectric and electromagnetic generator. The device is completely eco-friendly without any harmful effects.

The present work describes the TENG-EMG hybrid generator which works simultaneously upon the same sliding motions. The device was made of a polyethylene terephthalate (PET) cylinder housing composed of kapton film with gold-coated electrodes placed at the inner side and made as a negative triboelectric layer and paper bits as a positive triboelectric layer. The triboelectric series chart showing various triboelectric materials with their respective charges in Additional file 1: Figure S1. The outer side of the PET was wound with a copper coil and magnet is placed inside to generate EMG output. The TENG component generates more voltage and less current, whereas the EMG component generates low voltage and high current. By hybridizing both the harvesters using proper load matching resistance and rectification circuit the output power had been boosted and eventually been used for real-time demonstration such as charging the commercial capacitor and lighting up light-emitting diodes (LEDs). Further, the hybrid device had been used for scavenging small-scale biomechanical energy by placing the device on the backpack and been generated electrical output under the walking, running and bending motions of the human body. This type of hybrid energy harvester with enhanced output power can be used successively for self-powered sensors/systems and can be used for micro or nano-electromechanical systems (MEMS/NEMS) based applications.

\section{Device fabrication and measurements}

A cylindrical housing made of PET had been used as the outer layer for the hybrid device (length is $50 \mathrm{~mm}$ and diameter is $20 \mathrm{~mm}$ ). A Kapton film was cut according to the size of the PET and coated with aluminum (Al) at the backside as the electrode material. The Kapton layer was kept as the inner wall and also acts as the negative triboelectric layer. A copper coil of 500 turns has been wounded outside the cylinder for the EMG component. Further neodymium magnet $\left(1 / 2^{\prime \prime}\right.$ diameter and $1 / 4^{\prime \prime}$ thick) and small bits of paper had placed inside the cylinder, where the paper acts as positive triboelectric material and magnet for the production of electromagnetic induction.

The electrical measurements were carried out with the help of an electrometer (Keithley 6514), and the linear vibration to the device for the measuring the electrical output was given using a linear motor (Lin Mot, Inc, USA). All measurements were carried out in room temperature inside a homemade Faraday cage for avoiding any external interference.

\section{Results and discussion}

Figure 1a shows the step by step schematic of the fabrication of the TENG-EMG hybrid generator device. The cylindrical housing made of PET with a dimension of $\mathrm{L}=50 \mathrm{~mm}$ and $\Phi=20 \mathrm{~mm}$. Kapton film with aluminum electrode coating was placed as the inner wall of the PET cylinder and eventually been used as a negative triboelectric layer. The outer wall of the cylinder was wounded by a copper coil with a maximum of 500 turns towards the fabrication of EMG component. Small square-shaped papers were cut and placed inside the cylinder, which acts as a positive triboelectric material and a lightweight neodymium magnet as shown in Fig. 1c is placed inside for the production of electromagnetic induction through the coil. This type of arrangement in the device can make the simultaneous generation of electric power from both EMG and TENG independently upon the same mechanical vibration. Figure $1 \mathrm{~b}$ shows the comparison of the use of the TENG-EMG hybrid device over the usage of battery. It is well known that the battery has certain 


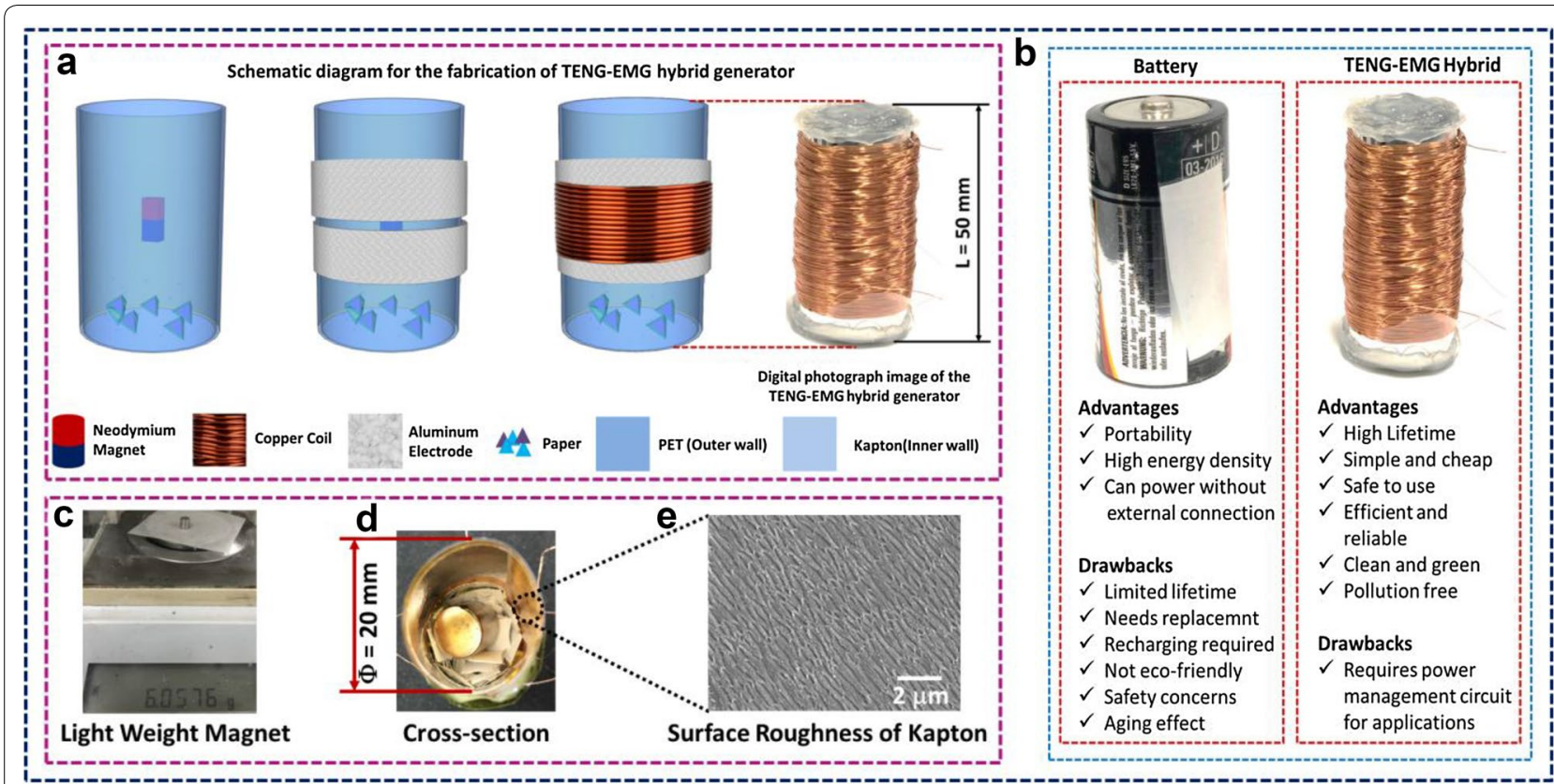

Fig. 1 a Schematic diagram of the fabrication of TENG-EMG hybrid device. $\mathbf{b}$ Comparison of the performance of TENG-EMG hybrid device and commercial battery with their advantages and disadvantages. c Digital photograph image of the magnet used and its weight, $\mathbf{d}$ cross-section of the device showing the magnet and paper flakes with dimension and $\mathbf{e}$ FE-SEM image showing the surface roughness created on Kapton film using plasma etching process

drawbacks such as limited lifetime and requires periodical replacement. Rechargeable batteries require external power for recharging it for its continuous usage. With the time passing the battery performance can be affected due to its aging effect. The safety about the battery is still a problem because chemical energy has been converted into electrical energy and it is not eco-friendly. These drawbacks can be overcome easily by using the TENGEMG hybrid device. The only drawback which these type of devices face is that it requires an external power management circuit to deliver the stable electrical output for using it into any real-time applications. Figure $1 \mathrm{~d}$ shows the cross-section of the device fabricated, showing the paper flakes and magnet. The Kapton film used here is a micro-roughness created film using a plasma etching process. The micro-roughness enhances the number of contact point $\mathrm{s}$ on the layers leading to the development of high electrical output. The roughness profile was measured using a field emission scanning electron microscope (FE-SEM) and is shown in Fig. 1e.

Figure 2 describes the working mechanism of both triboelectric nanogenerator and electromagnetic generator upon the sliding mode. Figure 2a shows the working mechanism of sliding mode TENG, in which the paper slides on the kapton film. Under linear vibration applied on the device, the papers inside the devices act as a positive triboelectric layer that moves over the negative kapton surface, leads to the generation of electric potential. This makes the electron to flow from one electrode to the other through an external load resistance as shown in Fig. 2a(ii). Figure 2a(iii) shows the papers were back to the normal position and makes to complete one halfcycle of the TENG component with no flow of electrons. Next, with the second half cycle, the paper moves on the opposite direction make the electrons to flow in the reverse direction as shown in Fig. 2b(iv). Figure (c) and (d) shows the triboelectric output voltage and current with the maximum electrical output of open-circuit voltage of $20 \mathrm{~V}$ and short circuit current of $300 \mathrm{nA}$ peak to peak. Similarly, the working mechanism of EMG was shown in Fig. 2b(i-iv). When a linear vibration is applied to the device, the magnet inside the cylinder moves across the coil leads to the production of magnetic flux around the coil which pushes electrons in the copper wire. The electrons mobility is directly responsible for the electric charge flow which was determined through Lenz's law. The generated output voltage and current from the EMG component can be calculated using Faraday's law. Figure 2e, $f$ shows the electrical output of the EMG with the maximum peak to peak voltage $\approx 2 \mathrm{~V}$ and a current of $10 \mathrm{~mA}$ at an operating frequency of $2 \mathrm{~Hz}$.

Figure $3 \mathrm{a}, \mathrm{b}$ shows the force analysis of the triboelectric component. The voltage and current response have been analyzed by applying the linear acceleration ranging $1 \mathrm{~m} /$ 


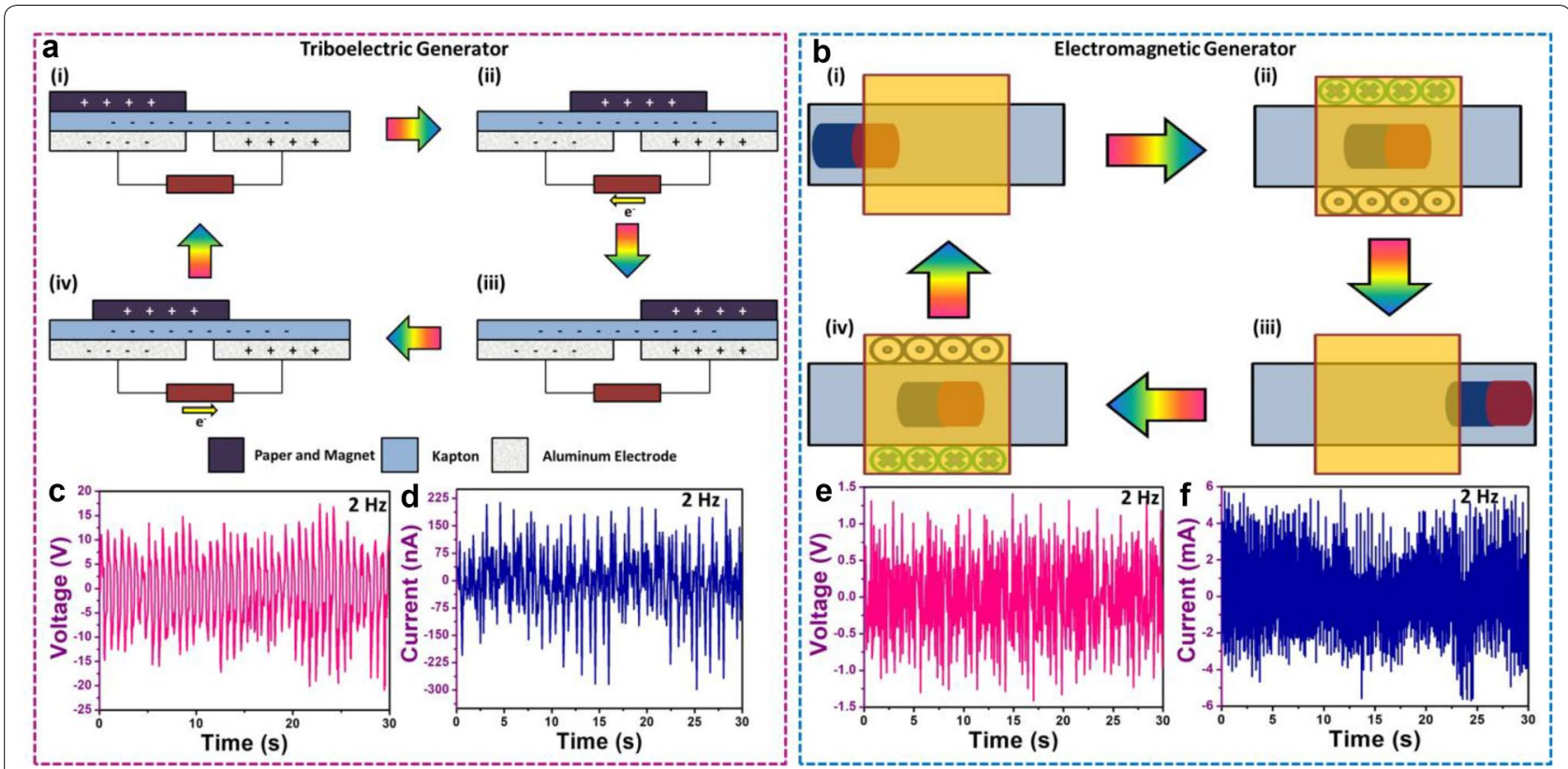

Fig. 2 a Working mechanism of the triboelectric generator under sliding motion. $\mathbf{b}$ Working mechanism of the electromagnetic generator, $\mathbf{c}, \mathbf{d}$ voltage and current output of TENG component, $\mathbf{e}$, f voltage and current output of EMG component

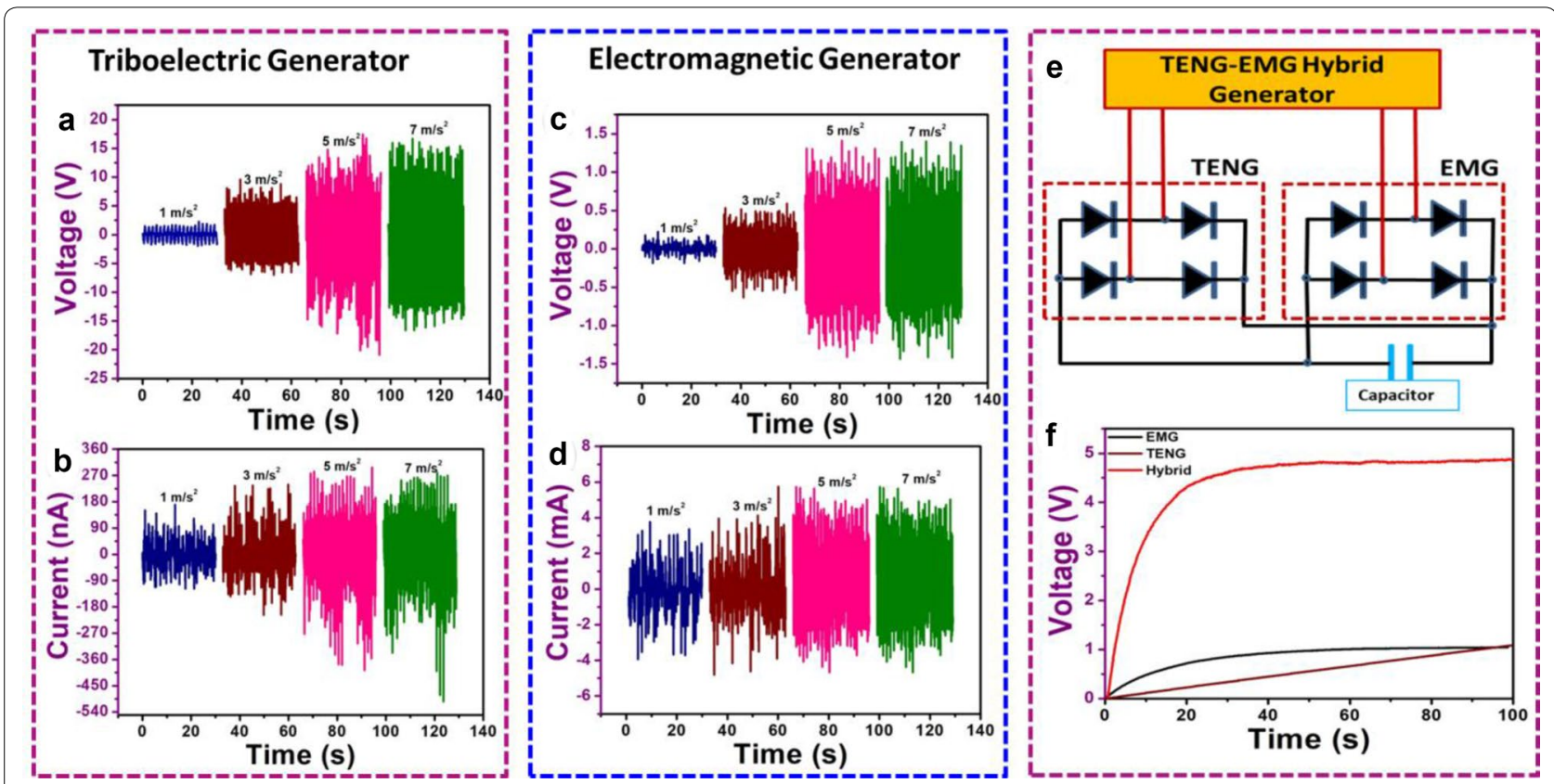

Fig. 3 a, $\mathbf{b}$ Force analysis of TENG component with different acceleration ranging from ranging $1 \mathrm{~m} / \mathrm{s}^{2}, 3 \mathrm{~m} / \mathrm{s}^{2}, 5 \mathrm{~m} / \mathrm{s}^{2}$ and $7 \mathrm{~m} / \mathrm{s}^{2}$. $\mathbf{c}$ Load resistance analysis of TENG component, $\mathbf{d}$, e Force analysis of EMG component under various acceleration from ranging $1 \mathrm{~m} / \mathrm{s}^{2}, 3 \mathrm{~m} / \mathrm{s}^{2}, 5 \mathrm{~m} / \mathrm{s}^{2}$ and $7 \mathrm{~m} /$ $s^{2}$. $\mathbf{L}$ Load resistance analysis of EMG component, $\mathbf{g}$ circuit connection diagram used for charging commercial capacitor. $\mathbf{h}$ Capacitor charging characteristics by TENG, EMG and hybrid combination

$\mathrm{s}^{2}, 3 \mathrm{~m} / \mathrm{s}^{2}, 5 \mathrm{~m} / \mathrm{s}^{2}$ and $7 \mathrm{~m} / \mathrm{s}^{2}$. The output gets stabilized once the acceleration reaches $5 \mathrm{~m} / \mathrm{s}^{2}$, with the further increase in the acceleration to $7 \mathrm{~m} / \mathrm{s}^{2}$ the output response gets stabilized. This is due to the volume of the cylinder used as well the quantity of the paper used. From this, the maximum acceleration used was confirmed to be $5 \mathrm{~m} / \mathrm{s}^{2}$, 
and the output voltage generated was $20 \mathrm{~V}$ and the maximum current of $300 \mathrm{nA}$. On the other hand, the force analysis was performed for the EMG component with the same acceleration used for the TENG component as shown in Fig. 3c, d. Similar to the TENG component the output of EMG component also saturates at $5 \mathrm{~m} / \mathrm{s}^{2}$ acceleration, showing the maximum output voltage of $2 \mathrm{~V}$ and a current of $10 \mathrm{~mA}$. Figure $3 \mathrm{e}$ shows the circuit diagram used for charging the commercial capacitor of 2.2 $\mu \mathrm{F}$ under TENG, EMG, and hybrid configurations. The charging characteristics of a commercial capacitor using the hybrid connection charges rapidly when compared to individual TENG and EMG components. This behavior is due to the higher output under hybrid connection, whereas in the individual TENG the voltage is higher and current is too low to trigger the charging phenomenon. The same behavior in charging occurs for the individual EMG, where the voltage is less to induce the charging as shown in Fig. $3 f$.
The load matching analysis was performed by using different load resistance, and the TENG component shows the instantaneous peak power of $1 \mu \mathrm{W}$ and the power density $1.2 \mathrm{~mW} / \mathrm{m}^{2}$ at a load resistance of $500 \mathrm{M} \Omega$ shown in Additional file 1: Figure S2. The EMG component shows the peak power of $4 \mathrm{~mW}$ at $5 \mathrm{k} \Omega$ load resistance as shown in Fig. 4a, c. The voltage response of both TENG and EMG device was concerning change in frequency is shown in Fig. 4b, d. The device was mounted on an electro-dynamic shaker and measured its voltage response for different frequency. The displacement of the shaft is also a factor considered during measurement. So, the displacement is considered from $2 \mathrm{~mm}, 4 \mathrm{~mm}, 6 \mathrm{~mm}, 8 \mathrm{~mm}$ and $10 \mathrm{~mm}$ and the respected change in frequency response have also been measured. The durability of the fabricated device was shown in Fig. 5a, b for 2000s by analyzing the voltage (TENG) and current (EMG). Figure 5c shows the demonstration of lighting up high power white LEDs with

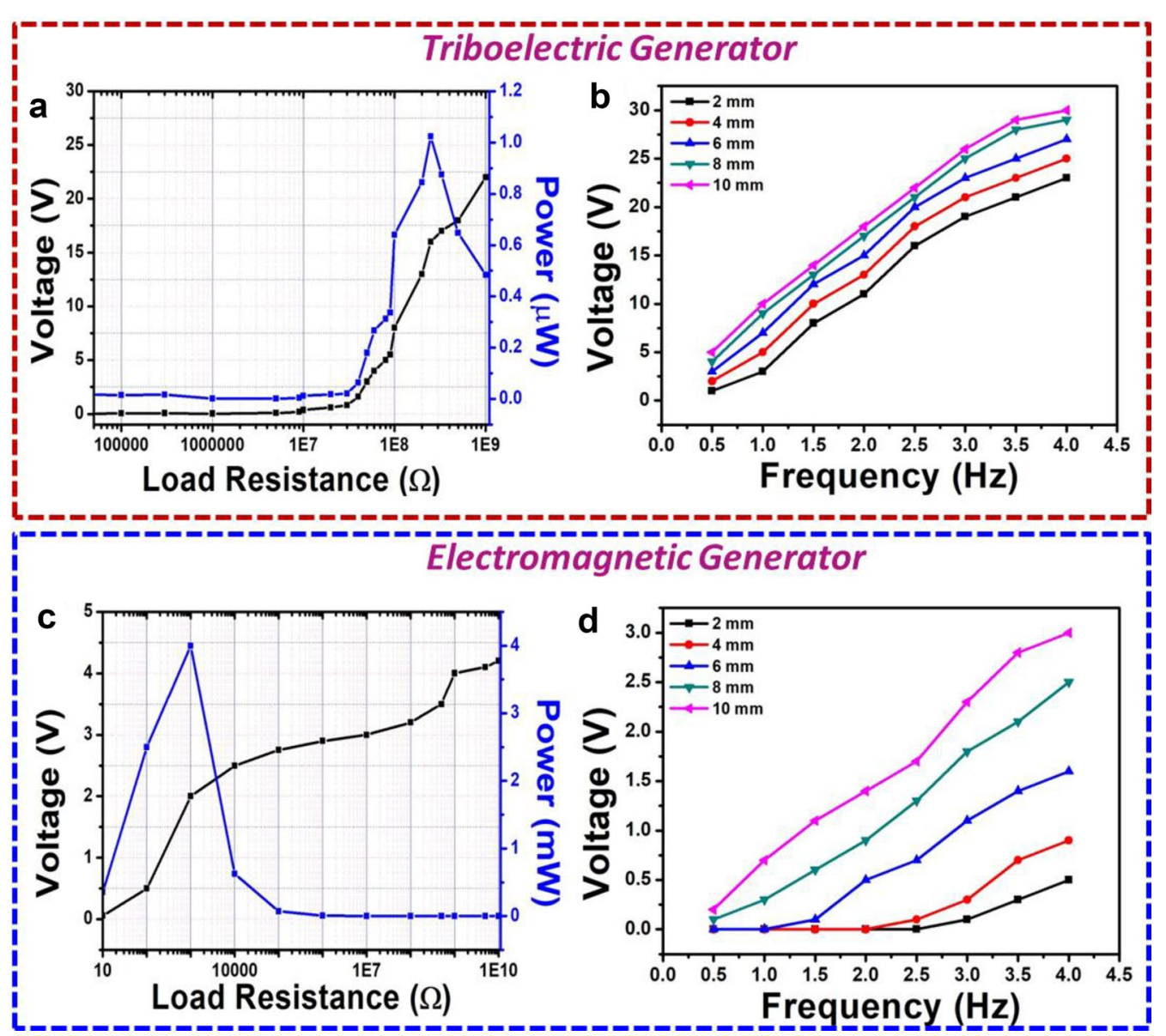

Fig. 4 a Load resistance analysis of the TENG component and its instantaneous power value, $\mathbf{b}$ voltage measurement of the TENG component with respect to different frequency, $\mathbf{c}$ load resistance and power analysis of the EMG component, $\mathbf{d}$ voltage measurement of EMG component with respect to the different operating frequency 

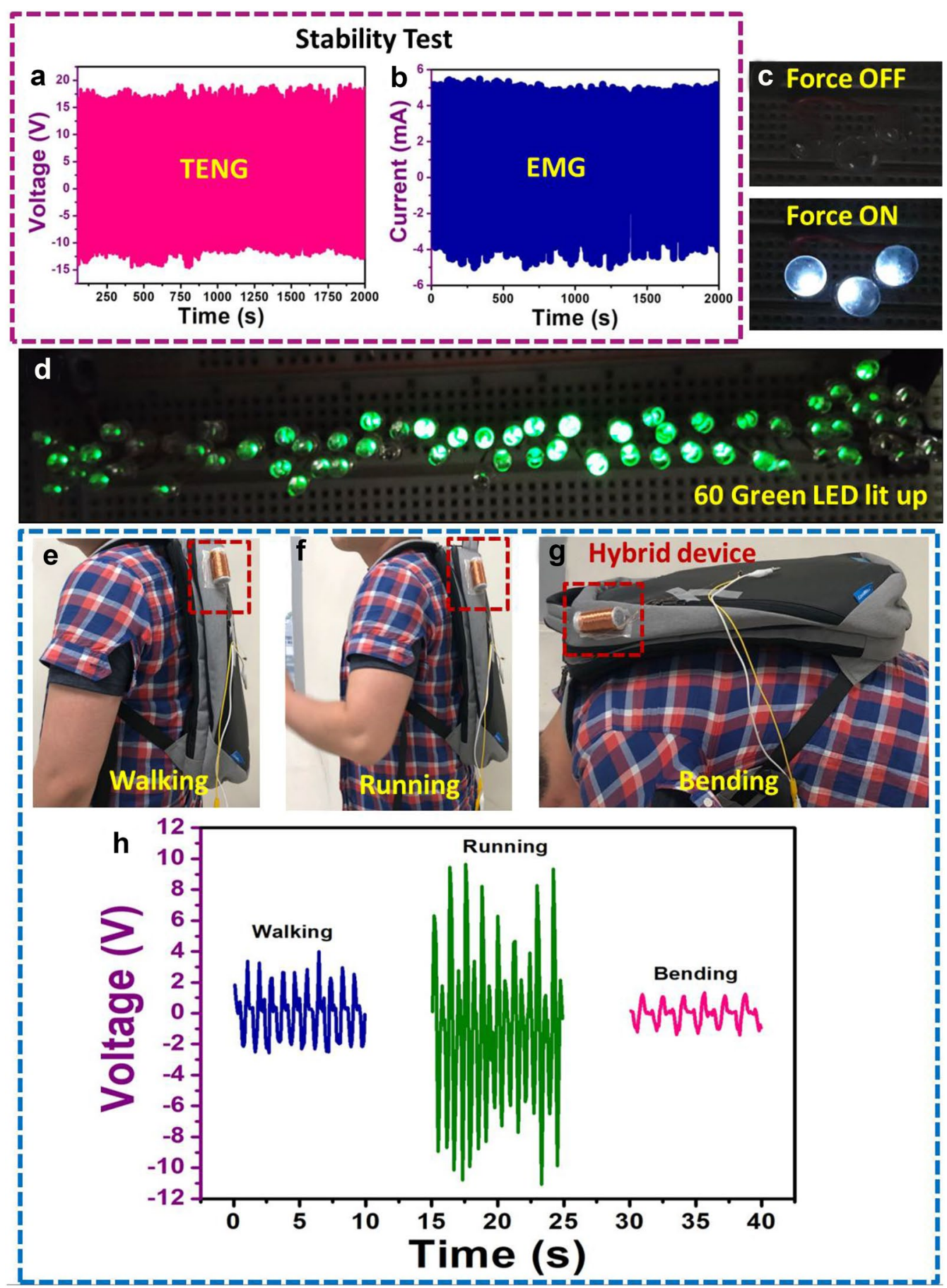

Fig. 5 a, b Stability analysis of TENG and EMG component. c High power White LEDs lit up under hybrid connection, $\mathbf{d} 60$ green LED lit up using hybrid device $\mathbf{e}-\mathbf{g}$ real time biomechanical energy harvesting by attaching the device on a backpack and analysis of electrical output by walking, running and bending motions, $\mathbf{h}$ electrical output generated under walking, running and bending

force ON and OFF to the device. Further 60 green LEDs were also lit up using the hybrid configuration which is shown in Fig. 5. The hybrid device had been used for the real-time demonstration of small-scale bio-mechanical energy scavenging by placing the device on a backpack. Both the EMG and TENG output connections 
have been connected in series for the real-time human motion data response acquisition. Figure $5 \mathrm{e}-\mathrm{g}$ shows the device attached on the backpack and could scavenge the biomechanical energy through walking, running and bending motion of the human wearing the bag. The real-time electrical output of the hybrid device (TENGEMG connected in series) on scavenging the biomechanical energy is shown in Fig. $5 \mathrm{~h}$. The higher output was obtained from the running motion which is due to the high acceleration applied to the device during a running motion. Similarly, the output is low during walking and bending. The change in output is directly related to the triboelectric component (i.e., paper) which moves according to the force applied on to the device. The phenomenon can be understood directly from the peak pattern in the electrical signal during the real-time analysis. Hence the above results clearly show that the TENG-EMG hybrid device is a promising candidate for scavenging small-scale biomechanical energy and can to charge the commercial capacitor and lighting up LEDs. With further development in this field can make a potential breakthrough in the field of MEMS and NEMS systems as well as self-powered sensors and systems.

\section{Conclusions}

In summary, a single package hybrid generator composed of TENG and EMG had reported via a cost-effective and easy fabrication method. The device works actively upon various accelerations and generates electrical output simultaneously from both TENG and EMG components upon the same motion. The working mechanism of the individual components have been discussed, and the electrical analysis had performed extensively under various accelerations, load matching analysis, capacitor charging, and LED lit up. The device generates a maximum electrical output of $20 \mathrm{~V}$ and $300 \mathrm{nA}$ current at an acceleration of $5 \mathrm{~m} / \mathrm{s}^{2}$ with an instantaneous power density of $1 \mathrm{~mW} / \mathrm{m}^{3}$ for the TENG component. Similarly, the EMG component generates a maximum output with the voltage of $2 \mathrm{~V}$ and a current of $10 \mathrm{~mA}$ at $5 \mathrm{~m} / \mathrm{s}^{2}$ with an instantaneous power $\approx 4 \mathrm{~mW}$ for EMG and $1 \mu \mathrm{W}$ for TENG components. Further, the hybrid device has been used for demonstrating the real-time application of small-scale biomechanical energy scavenging by attaching the device on the backpack and harvest energy from human walking, running and bending motions. From the above results, the hybrid energy harvesting technology using TENG and EMG paves the way for smart self-powered sensors and systems.

\section{Supplementary information}

Supplementary information accompanies this paper at https://doi. org/10.1186/s40486-019-0093-6.

Additional file 1: Figure S1. Triboelectric series chart showing the triboelectric positive and negative charges. Figure S2. Load resistance and power density analysis of the TENG component in the hybrid generator device.

\section{Abbreviations}

TENG: triboelectric nanogenerator; EMG: electromagnetic generator; PET: polyethylene terephthalate; MEMS: micro electro mechanical systems; NEMS: nano electro-mechanical systems; LED: light-emitting diode; PENG: piezoelectric nanogenerator.

\section{Acknowledgements}

The authors thank Dr. Arunkumar Chandrasekhar for providing valuable suggestions on the application part.

\section{Authors' contributions}

W and WJK designed and fabricated the experiments. YP performs the electrical analysis of the device. KSA designed the figures and images. NRA and SJK participated in drafting the manuscript and co-coordinating for the overall experiments. All authors read and approved the final manuscript.

\section{Funding}

This research was supported by the 2019 scientific promotion program funded by Jeju National University.

Availability of data and materials

All data produced and analyzed in this study are included in this article.

Competing interests

The authors declare that they have no competing interests.

Received: 15 May 2019 Accepted: 30 September 2019

Published online: 05 October 2019

\section{References}

1. Wang S, Wang X, Wang ZL, Yang Y (2016) Efficient scavenging of solar and wind energies in a smart city. ACS Nano 10(6):5696-5700

2. Vivekananthan V, Alluri NR, Purusothaman Y, Chandrasekhar A, Kim S-J (2017) A flexible, planar energy harvesting device for scavenging road side waste mechanical energy via the synergistic piezoelectric response of K0.5 Na0.5 NbO3-BaTiO3/PVDF composite films. Nanoscale. 9(39):15122-15130

3. Vivekananthan V, Alluri NR, Chandrasekhar A, Purusothaman Y, Gupta A, Kim S-J (2019) Zero-power consuming intruder identification system by enhanced piezoelectricity of $\mathrm{K} 0.5 \mathrm{NaO} .5 \mathrm{NbO} 3$ using substitutional doping of BTO NPs. J Mater Chem C. 7:7563

4. Wen Z, Guo H, Zi Y, Yeh MH, Wang X, Deng J et al (2016) Harvesting broad frequency band blue energy by a triboelectric-electromagnetic hybrid nanogenerator. ACS Nano 10(7):6526-6534

5. Li DJ, Hong S, Gu S, Choi Y, Nakhmanson S, Heinonen O et al (2014) Polymer piezoelectric energy harvesters for low wind speed. Appl Phys Lett 104(1):012902

6. Lee S, Bae S-H, Lin L, Yang Y, Park C, Kim S-W et al (2013) Super-flexible nanogenerator for energy harvesting from gentle wind and as an active deformation sensor. Adv Func Mater 23(19):2445-2449

7. Hinchet R, Kim S-W (2015) Wearable and implantable mechanical energy harvesters for self-powered biomedical systems. ACS Nano 9(8):7742-7745 
8. Vivekananthan V, Chandrasekhar A, Alluri NR, Purusothaman Y, Joong Kim W, Kang C-N et al (2019) A flexible piezoelectric composite nanogenerator based on doping enhanced lead-free nanoparticles. Mater Lett 249:73-76

9. Wang ZL, Song J (2006) Piezoelectric nanogenerators based on zinc oxide nanowire arrays. Science 312(5771):242-246

10. Pan R, Xuan W, Chen J, Dong S, Jin H, Wang X et al (2018) Fully biodegradable triboelectric nanogenerators based on electrospun polylactic acid and nanostructured gelatin films. Nano Energy. 45:193-202

11. Quan T, Wang X, Wang ZL, Yang Y (2015) Hybridized electromagnetictriboelectric nanogenerator for a self-powered electronic watch. ACS Nano 9(12):12301-12310

12. Chandrasekhar A, Alluri NR, Vivekananthan V, Park JH, Kim S-J (2017) Sustainable biomechanical energy scavenger toward self-reliant kids' interactive battery-free smart puzzle. ACS Sustain Chem Eng 5(8):7310-7316

13. Zhao Z, Pu X, Du C, Li L, Jiang C, Hu W et al (2016) Freestanding flag-type triboelectric nanogenerator for harvesting high-altitude wind energy from arbitrary directions. ACS Nano 10(2):1780-1787

14. Yao Y, Jiang T, Zhang L, Chen X, Gao Z, Wang ZL (2016) Charging system optimization of triboelectric nanogenerator for water wave energy harvesting and storage. ACS Appl Mater Interfaces 8(33):21398-21406

15. Zhang B, Chen J, Jin L, Deng W, Zhang L, Zhang H et al (2016) Rotatingdisk-based hybridized electromagnetic-triboelectric nanogenerator for sustainably powering wireless traffic volume sensors. ACS Nano 10(6):6241-6247
16. Lei R, Zhai H, Nie J, Zhong W, Bai Y, Liang X et al (2019) Butterfly-inspired triboelectric nanogenerators with spring-assisted linkage structure for water wave energy harvesting. Adv Mater Technol 4(3):1800514

17. Fan X, Chen J, Yang J, Bai P, Li Z, Wang ZL (2015) Ultrathin, rollable, paperbased triboelectric nanogenerator for acoustic energy harvesting and self-powered sound recording. ACS Nano 9(4):4236-4243

18. Quan T, Wang ZL, Yang Y (2016) A shared-electrode-based hybridized electromagnetic-triboelectric nanogenerator. ACS Appl Mater Interfaces 8(30):19573-19578

19. Vivekananthan $V$, Chandrasekhar A, Alluri NR, Purusothaman $Y$, Khandelwal G, Pandey R et al (2019) $\mathrm{Fe}_{2} \mathrm{O}_{3}$ magnetic particles derived triboelectric-electromagnetic hybrid generator for zero-power consuming seismic detection. Nano Energy. 64:103926

20. He J, Wen T, Qian S, Zhang Z, Tian Z, Zhu J et al (2018) Triboelectricpiezoelectric-electromagnetic hybrid nanogenerator for high-efficient vibration energy harvesting and self-powered wireless monitoring system. Nano Energy. 43:326-339

21. Huiyun S, Ping C, Ruixuan C, Lingjie X, Na S, Qingqing S et al (2018) Triboelectric-electromagnetic hybrid generator for harvesting blue energy. Nano-Micro Lett 10(3):54

\section{Publisher's Note}

Springer Nature remains neutral with regard to jurisdictional claims in published maps and institutional affiliations.

\section{Submit your manuscript to a SpringerOpen ${ }^{\circ}$ journal and benefit from:}

- Convenient online submission

$\checkmark$ Rigorous peer review

- Open access: articles freely available online

- High visibility within the field

- Retaining the copyright to your article

Submit your next manuscript at $\boldsymbol{\nabla}$ springeropen.com 